\title{
Correction to: N-Glycoproteomics Study of Putative N-Glycoprotein Biomarkers of Drug Resistance in MCF-7/ADR Cells657
}

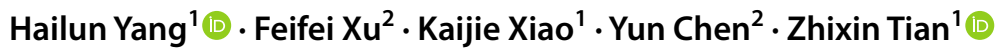

(c) International Human Phenome Institutes (Shanghai) 2022

\section{Correction to: Phenomics} https://doi.org/10.1007/s43657-021-00029-8

For Fig. 3, Fig. 3a, b has been mistakenly mixed with the same image, for Fig. 5, there should be 5 green dots in
Fig. 5a instead of 6 green dots. "Discussion" should be "Discussion". The figures should have appeared as shown below:

The original article can be found online at https://doi.org/10.1007/ s43657-021-00029-8.

Yun Chen

ychen@njmu.edu.cn

$\bowtie$ Zhixin Tian

zhixintian@tongji.edu.cn

1 Shanghai Key Laboratory of Chemical Assessment and Sustainability, School of Chemical Science and Engineering, Tongji University, Shanghai 200092, China

2 School of Pharmacy, Nanjing Medical University, Nanjing 211166, China 

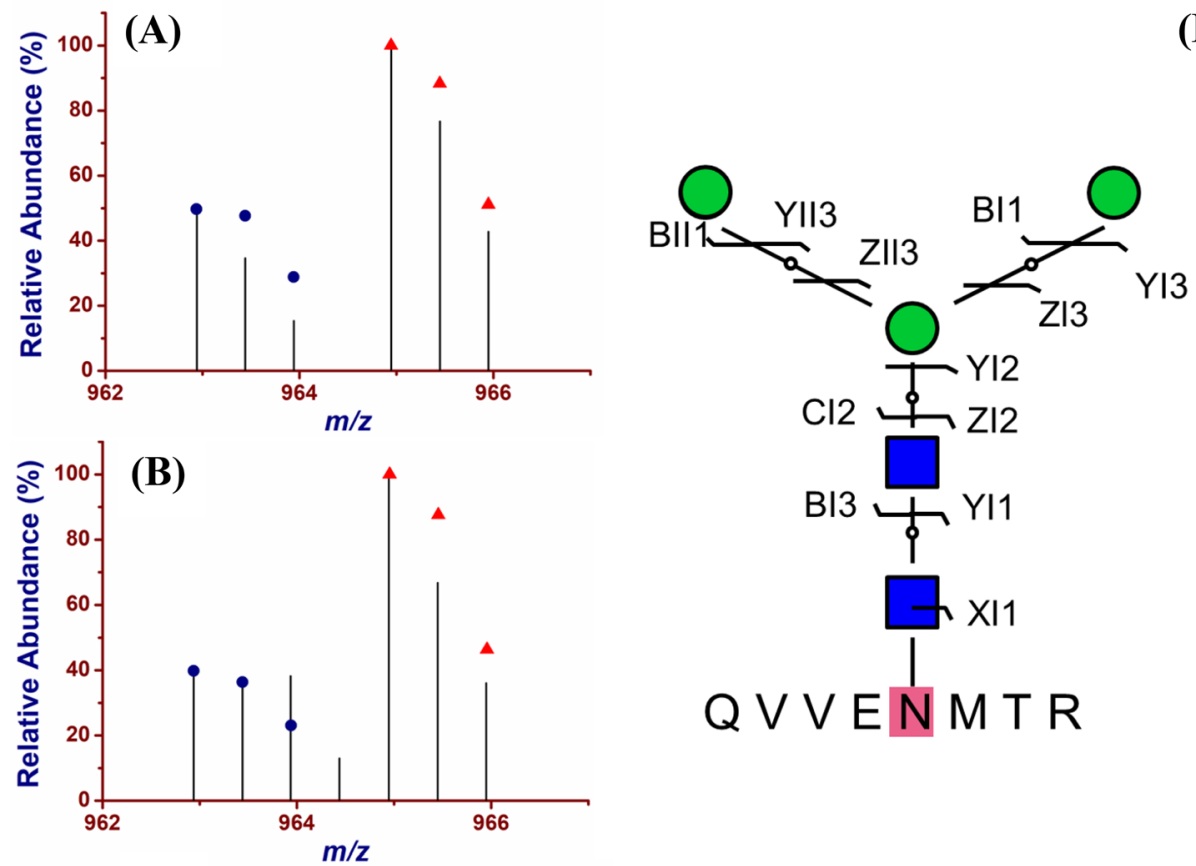

(D)

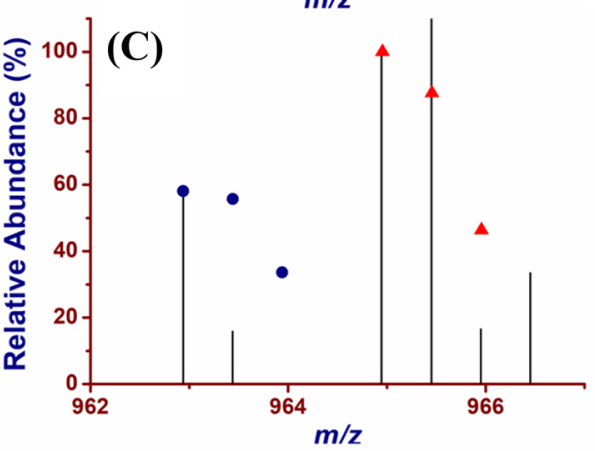

(E)

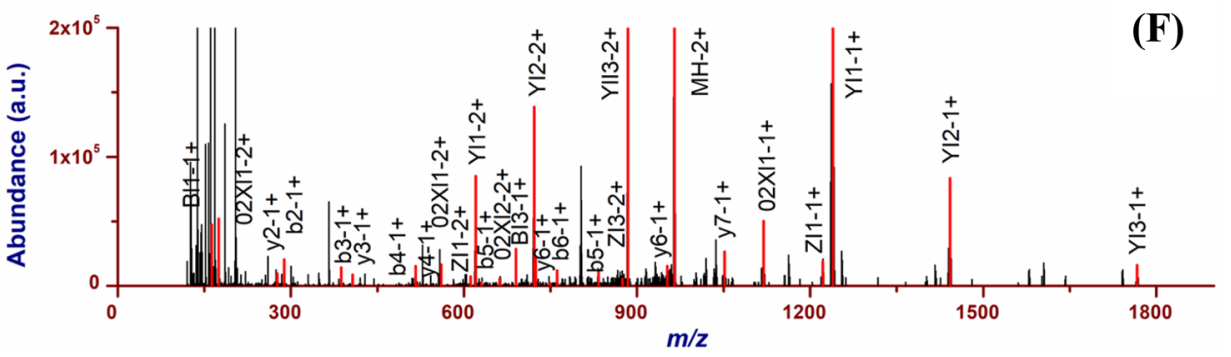




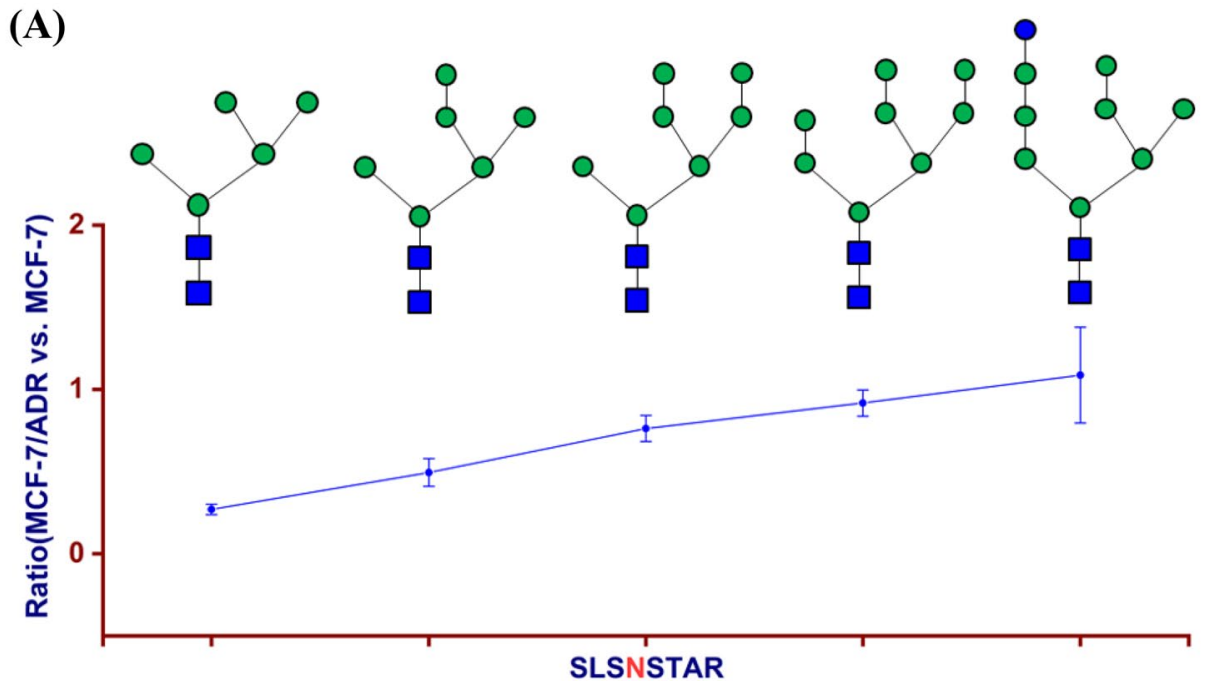

(B)

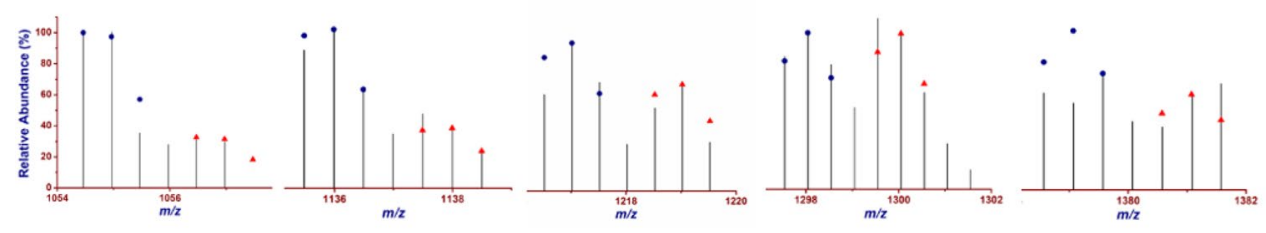

The original article has been updated. 